\title{
Mutational analysis of the BRAF, RAS and EGFR genes in human adrenocortical carcinomas
}

\author{
Vassiliki Kotoula, Elias Sozopoulos ${ }^{1}$, Helen Litsiou ${ }^{1}$, Galinos Fanourakis ${ }^{1}$, \\ Triantafyllia Koletsa, Gerassimos Voutsinas ${ }_{5}^{2}$, Sophia Tseleni-Balafouta ${ }^{1}$, \\ Constantine S Mitsiades ${ }^{3,4}$, Axel Wellmann ${ }^{5}$ and Nicholas Mitsiades ${ }^{3,4,6}$
}

\footnotetext{
Department of Pathology, School of Medicine, Aristotle University of Thessaloniki, University Campus, Thessaloniki 54006, Greece ${ }^{1}$ Department of Pathology, University of Athens, Athens, Greece

'Laboratory of Environmental Mutagenesis and Carcinogenesis, Institute of Biology, NCSR 'Demokritos', GR-15310 Athens, Greece

${ }^{3}$ Department of Medical Oncology, Harvard Medical School, Dana Farber Cancer Institute, Boston, Massachusetts 02115, USA

${ }^{4}$ Department of Medicine, Harvard Medical School, Boston, Massachusetts 02115, USA

${ }^{5}$ Institute of Pathology, University Clinic, RWTH Aachen, 52074 Aachen, Germany

${ }^{6}$ Memorial Sloan-Kettering Cancer Center, New York, New York 10065, USA

(Correspondence should be addressed to N Mitsiades; Email: mitsiadn@mskcc.org; V Kotoula; Email: vkotoula@auth.gr)
}

\begin{abstract}
The serine/threonine kinase B-Raf plays a key role in the Ras/Raf/MEK/ERK pathway that relays extracellular signals for cell proliferation and survival. Several types of human malignancies harbor activating $B R A F$ mutations, most frequently a V600E substitution. The epidermal growth factor receptor (EGFR), a transmembrane tyrosine kinase (TK) receptor that mediates proliferation and survival signaling, is expressed in a wide variety of normal and neoplastic tissues. EGFR inhibitors have produced objective responses in patients with non-small cell lung carcinomas harboring activating EGFR TK domain somatic mutations. We evaluated the presence of mutations in BRAF (exons 11 and 15), KRAS (exons 1 and 2), NRAS (exons 1 and 2), and EGFR (exons 18-21) in adrenal carcinomas (35 tumor specimens and two cell lines) by DNA sequencing. BRAF mutations were found in two carcinomas $(5.7 \%)$. Four carcinomas $(11.4 \%)$ carried EGFRTK domain mutations. One specimen carried a KRAS mutation, and another carried two NRAS mutations. No mutations were found in the two adrenocortical cell lines. BRAF- and EGFR-mutant tumor specimens exhibited stronger immunostaining for the phosphorylated forms of the MEK and ERK kinases than their wild-type counterparts. EGFR-mutant carcinomas exhibited increased phosphorylation of EGFR (Tyr 992) compared with wild-type carcinomas. We conclude that BRAF, RAS, and EGFR mutations occur in a subset of human adrenocortical carcinomas. Inhibitors of the Ras/Raf/MEK/ERK and EGFR pathways represent candidate targeted therapies for future clinical trials in carefully selected patients with adrenocortical carcinomas harboring respective activating mutations.
\end{abstract}

Endocrine-Related Cancer (2009) 16 565-572

\section{Introduction}

Adrenocortical carcinoma is a rare endocrine tumor with overall poor prognosis. Complete surgical resection is the only potentially curative treatment, but even then, distant recurrences are common. Adrenocortical carcinoma is resistant to conventional chemotherapeutics. Mitotane therapy may improve symptoms, but does not significantly alter survival. The genetic background of adrenocortical carcinoma is poorly understood, and more studies in this area are necessary in order to identify new targets for tailored therapies.

The Raf kinase family comprises A-Raf, B-Raf, and C-Raf (also called Raf-1) that play central roles in the conserved Ras/Raf/MEK/ERK pathway. The latter acts to relay signals from cell surface receptors, such as the epidermal growth factor receptor (EGFR, also known as HER1, a member of the HER family of 
transmembrane receptors), to the $\mathrm{p} 42 / \mathrm{p} 44$ MAP kinases (ERK1/2), the key effectors of this pathway. Activating mutations of $B R A F$ exons 11 and 15 occur commonly in human malignant melanomas and thyroid carcinomas, and, less frequently, in colorectal, lung, ovarian, breast and liver carcinomas, sarcomas, and gliomas (Davies et al. 2002, Pollock \& Meltzer 2002, Kimura et al. 2003, Garnett \& Marais 2004, Mitsiades et al. 2007). The most common mutation is a T1799A nucleotide change resulting in a V600E amino acid residue change within the B-Raf activation domain (Nikiforova et al. 2003), generating a protein with high baseline enzymatic activity that leads to constitutive ERK activation. Therefore, B-Raf represents a promising target for the development of novel anticancer therapies (Fagin 2004, Garnett \& Marais 2004, Mitsiades et al. 2007). The mutational status of $B R A F$ in adrenocortical carcinomas is presently unknown.

The EGFR pathway has also been proposed to be important for cancer pathophysiology, as it has been implicated in proliferation, migration, stromal invasion, tumor angiogenesis, and resistance to cell death-inducing signals. Somatic activating mutations within the EGFR tyrosine kinase (TK) domain are present in a subset of non-small cell lung cancers (NSCLCs; Lynch et al. 2004, Paez et al. 2004) and confer tumor cell sensitivity to inhibitors of the EGFR TK, such as gefitinib (Iressa, AstraZeneca Pharmaceuticals) and erlotinib (Tarceva, Genentech, San Francisco, CA, USA). EGFR is expressed in adrenocortical carcinomas (Kamio et al. 1990, Edgren et al. 1997), yet its mutational status in this malignancy is presently unknown.

In the present study, we investigated the mutational status of BRAF, KRAS, NRAS, and EGFR in adrenocortical carcinomas.

\section{Materials and methods}

\section{Human tissues}

Archival formalin-fixed and paraffin-embedded tumor specimens from 35 patients (16 females and 19 males) with adrenocortical carcinomas, ages 7-81 years (mean \pm s.D.: $53.2 \pm 14.2$ ), as well as five adrenal adenomas, were retrieved retrospectively from the files of the Institute of Pathology, University of Bonn, Germany, the Department of Pathology, School of Medicine, Aristotle University of Thessaloniki and the Department of Pathology, University of Athens, Greece, as well as from commercial sources (US Biomax, Rockville, MD, USA and Asterand, Detroit, MI, USA). All studies on patient material were conducted in accordance with the Declaration of
Helsinki principles and Institutional Review Board policies. To confirm the malignant nature of our specimens, we used the Weiss system that combines nine morphological parameters, including three structural ('dark' cytoplasm, diffuse architecture, necrosis), three cytological (atypia, mitotic count, atypical mitotic figures), and three related to invasion (of sinusoids, veins and tumor capsule; Volante et al. 2008). We also performed immunohistochemistry (see below) for Melan-A (adrenocortical marker) and TTF-1 (lung and thyroid marker), to confirm their adrenal origin and rule out the possibility of undetected metastasis from a lung carcinoma.

\section{Cell lines}

Two previously described adrenal carcinoma cell lines, NCI-H295 and SW-13 were purchased from the American Type Culture Collection (ATCC, Manassas, VA, USA) and cultured according to ATCC instructions.

\section{Direct sequencing}

Genomic DNA isolated from tissue specimens and cell lines was PCR-amplified using the following previously described primers: primer sequences for $K R A S$ (exons 1 and 2) and for NRAS (exons 1 and 2) were from Suzuki et al. (1990) for BRAF (exons 11 and 15) were from Brose et al. (2002) and for EGFR (exons 18, 19, 20, and 21, corresponding to the TK domain) were from Paez et al. (2004).

PCR reactions were performed using the following conditions: for $K R A S$ exon $1: 95^{\circ} \mathrm{C} \times 3 \mathrm{~min} ; 95^{\circ} \mathrm{C}$ $\times 40 \mathrm{~s}, 56^{\circ} \mathrm{C} \times 40 \mathrm{~s}, 72^{\circ} \mathrm{C} \times 40 \mathrm{~s}$, for 40 cycles; then $72^{\circ} \mathrm{C} \times 7 \mathrm{~min}$. For $K R A S$ exon 2 : $95^{\circ} \mathrm{C} \times 3 \mathrm{~min}$; $95^{\circ} \mathrm{C} \times 30 \mathrm{~s}, 55^{\circ} \mathrm{C} \times 30 \mathrm{~s}, 72^{\circ} \mathrm{C} \times 30 \mathrm{~s}$, for 40 cycles; then $72^{\circ} \mathrm{C} \times 7 \mathrm{~min}$. For both NRAS exons: $95^{\circ} \mathrm{C}$ $\times 3 \mathrm{~min} ; 95^{\circ} \mathrm{C} \times 40 \mathrm{~s}, 57^{\circ} \mathrm{C} \times 40 \mathrm{~s}, 72^{\circ} \mathrm{C} \times 40 \mathrm{~s}$, for 40 cycles; then $72^{\circ} \mathrm{C} \times 7 \mathrm{~min}$. For $B R A F$ exons 11 and 15, PCR conditions were as in Brose et al. (2002). For EGFR exons 18-21, PCR conditions were as in (Paez et al. 2004).

Cycle sequencing of the purified PCR products was carried out with one of the PCR primers using the Big dye terminator sequencing kit (Applied Biosystems, Foster City, CA, USA). The Sephadex G-50-purified cycle sequencing products were analyzed on an ABI PRISM 310 Genetic Analyzer (Applied Biosystems).

\section{Immunohistochemistry}

Immunohistochemistry was performed and evaluated as previously described (Mitsiades et al. 2006). The antibodies used to detect phospho-EGFR (Tyr992), 
phospho-Akt (Ser473), phospho-MEK, total MEK, phospho-MAPK p44/42 (ERK1/2), and total MAPK p44/42 were from Cell Signaling (Beverly, MA, USA); monoclonal antibody against thyroid transcription factor-1 was from Novocastra Laboratories Ltd (Newcastle upon Tyne, UK); and monoclonal antibody against Melan-A (Clone A103) was from Dako (Carpinteria, CA, USA). The peroxidase reaction was developed with DAB and the slides were counterstained with hematoxylin. Immunostaining was evaluated with a $20 \times$ lens by two expert pathologists (V K and $\mathrm{T} \mathrm{K}$ ) who were blinded to the mutational status of the tumors. A scoring system of negative $(<10 \%$ positive cells per tumor), faint ( $>50 \%$ faintly staining cells out of all positive ones per medium power fields (MPF)) and strong ( $>50 \%$ strongly staining cells out of all positive ones per MPF) was developed. At least 10 MPFs were evaluated per section, including the invasive front of the tumor as much as possible.

\section{Results}

\section{Histological and immunohistochemical confirmation of human adrenocortical carcinomas}

We confirmed the malignant nature of our specimens using the Weiss criteria, which combine nine morphological parameters, including three structural ('dark' cytoplasm, diffuse architecture, necrosis), three cytological (atypia, mitotic count, atypical mitotic figures) and three related to invasion (of sinusoids, veins and tumor capsule; Volante et al. 2008). We also documented immunohistochemically that all our adrenocortical carcinomas were strongly positive for Melan-A and negative for TTF-1 (not shown), thus confirming their adrenal origin and ruling out the possibility of undetected metastasis from a lung carcinoma.

\section{BRAF sequence analysis in adrenocortical carcinoma cells}

We sequenced BRAF exons 11 and 15 in DNA extracted from 35 adrenocortical carcinoma specimens and two cell lines. One specimen, from a 53-year-old female, carried a BRAF T1799A transversion, leading to a V600E amino acid substitution, which is the most common BRAF mutation described in cancers in general (Garnett \& Marais 2004). Another tumor specimen, from a 69-year-old female, carried a G1391A nucleotide transversion in BRAF exon 11, leading to a G464E amino acid substitution (Fig. 1A) in the highly conserved glycine-rich GXGXXG motif present within the kinase domain of B-Raf that frequently harbors somatic activating mutations (Garnett \& Marais 2004). This activating mutation has been previously described in ovarian (Davies et al. 2002) and colorectal (Rajagopalan et al. 2002) carcinomas, and in melanoma (Houben et al. 2004).

\section{KRAS and NRAS sequence analysis in adrenocortical carcinoma cells}

We also investigated the mutational status of $K R A S$ and NRAS in our panel. In one tumor specimen, we found a G34T transversion in KRAS exon 1, leading to substitution of cysteine for glycine in codon 12 (G12C). The codon 12 of KRAS is a well known hot spot, and the G12C substitution has been previously described in various other malignancies. Another specimen exhibited two NRAS exon 1 substitutions, a GTG $\rightarrow$ GCG $($ V8A) and a AAA $\rightarrow$ AAC $($ K16N). No other KRAS or NRAS mutations were found in our panel.

\section{EGFR sequence analysis in adrenocortical carcinoma cells}

We also investigated the mutational status of EGFR (exons 18-21) in our panel. We detected mutations in four carcinoma specimens (11.5\%). Specifically, a specimen from a 53-year-old woman (the same specimen carrying the above-mentioned $B R A F$ V600E mutation) also exhibited a G2193A transition in EGFR exon 19, that results in a premature stop at codon 731 (Fig. 1C). This mutation has been previously described in NSCLC (Huang et al. 2004).

Another carcinoma, from a 54-year-old woman, exhibited a T2168C transition in EGFR exon 18, resulting in a F723S amino acid substitution (Fig. 1E). Substitutions with serine have been reported at codons 721 and 724 in NSCLC (Tam et al. 2006, Ichihara et al. 2007).

Another carcinoma, from a 52-year-old woman, exhibited a C2354A transversion in EGFR exon 20, leading to a T785N amino acid substitution (Fig. 1G) and a G2569A transition in exon 21, leading to a G857R amino acid substitution (Fig. 1I). The latter mutation that affects a crucial residue in the activation loop of the EGFR kinase has been reported in colorectal cancer (Moroni et al. 2005) and lies next to codon 858, one of the most important 'hot-spots' for EGFR mutations in NSCLC. Specifically, the L858R substitution is frequently found in gefitinib and erlotinib responders in NSCLC.

Finally, a carcinoma from a 35-year-old male exhibited a G2533A transition in EGFR exon 21, 
A

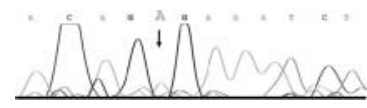

C

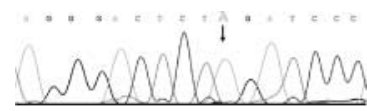

E

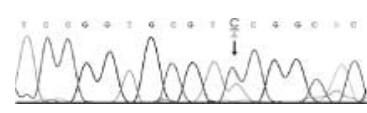

G

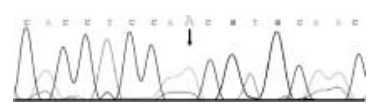

I

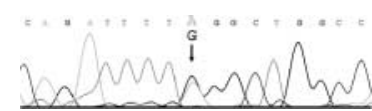

K

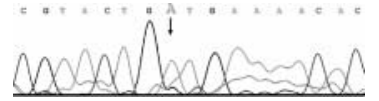

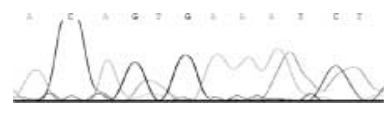

D

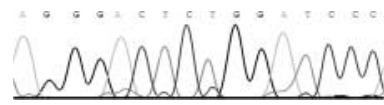

F

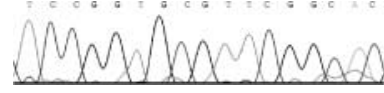

$\mathrm{H}$

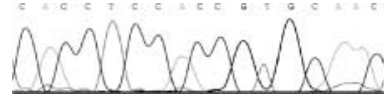

J

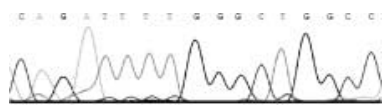

$\mathrm{L}$

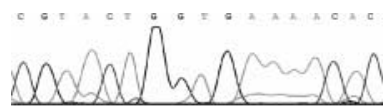

Glycine-rich Catalytic Activation

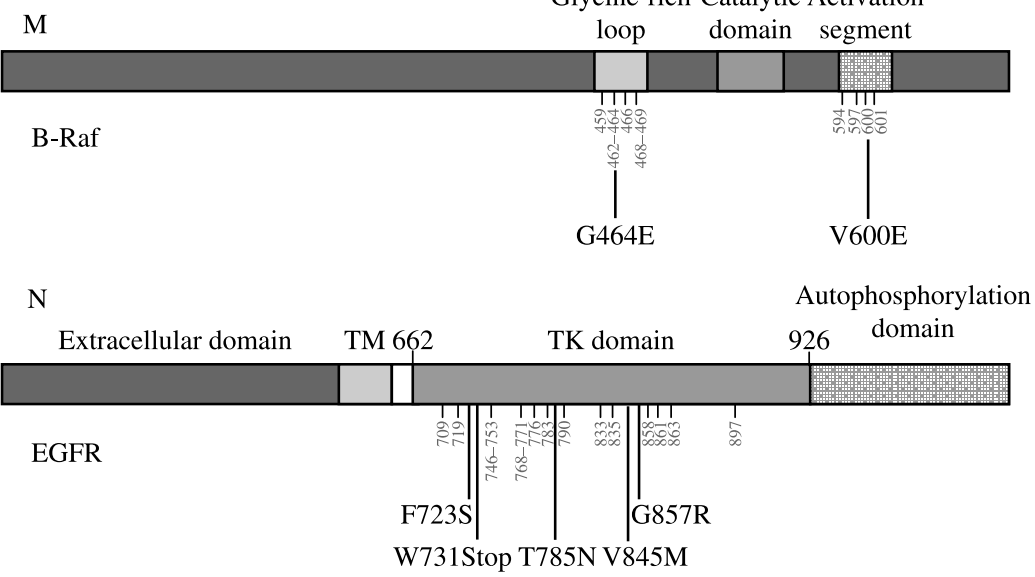

Figure 1 Sequence electropherograms of PCR product from adrenocortical carcinomas, depicting the presence of $B R A F$ or $E G F R$ mutations (indicated by the arrows). Specifically, (A). A T1799A transversion was detected in exon 15 of $B R A F$ in a carcinoma from a 53-year-old woman, leading to a V600E amino acid substitution. (B). The corresponding sequence from a wild-type carcinoma is depicted in. (C). The same tumor specimen as in (A) also exhibited a G2193A transition in exon 19 of EGFR, that results in a premature stop at codon 731. (D). The corresponding sequence from a wild-type carcinoma is depicted. (E). A carcinoma from a 54-year-old woman exhibited a T2168C transition in exon 18 of EGFR, resulting in a F723S amino acid substitution.

(F). The corresponding sequence from a wild-type carcinoma is depicted. A carcinoma from a 52-year-old woman exhibited a C2354A transversion in exon 20 of EGFR (G, leading to a T785N amino acid substitution) and a G2569A transition in exon 21 (I, leading to a G857R amino acid substitution). The corresponding sequence from a wild-type carcinoma is depicted in $(\mathrm{H})$ and $(\mathrm{J})$ respectively. (K). A carcinoma from a 35-year-old male exhibited a G2533A transition in exon 21 of EGFR, leading to a V845M amino acid substitution. The corresponding sequence from a wild-type carcinoma is depicted in (L). (M). Schematic diagram of $B R A F$ gene, depicting the glycine-rich loop, the catalytic domain and the activation segment, and the two mutations (G464E and V600E) found in our panel of adrenocortical carcinomas. Light grey numbers indicate sites of previously published $B R A F$ mutations. (N). Schematic diagram of EGFR gene, depicting the extracellular, transmembrane (TM), tyrosine kinase (TK, amino acid residues 662-926) and autophosphorylation domain and the mutations found in our panel of adrenocortical carcinomas. Light grey numbers indicate sites of previously published EGFR mutations. 
leading to a V845M amino acid substitution (Fig. 1K). No other EGFR mutations were found in our panel.

The location of these mutations on the $B R A F$ and $E G F R$ gene, as well as the location of previously described mutation 'hot-spots' in these genes is presented schematically in Fig. 1M and $\mathrm{N}$ respectively.

\section{Evidence for increased activation of the EGFR pathway in adrenocortical carcinoma cells har- boring mutations of EGFR}

We next evaluated the impact of EGFR mutations on the phosphorylation of this receptor. We found increased phospho-Tyr 992 immunostaining in carcinomas harboring EGFR mutations, compared with wild-type carcinomas (Fig. 2A and B).

\section{Evidence for increased activation of the MEK/ERK pathway in adrenocortical carcinoma cells harboring mutations of BRAF or EGFR}

We next evaluated the impact of $B R A F$ and $E G F R$ mutations on the activation of downstream signaling effectors, such as MEK and p42/44 MAPK (ERK1/2), which are activated via phosphorylation. Carcinomas that harbored a mutation in either gene exhibited increased phosphorylation of these kinases compared with carcinomas that were wild-type for both genes (Fig. 2C-H). However, we did not find increased immunostaining for phosphorylated Akt (Ser473) in the mutant carcinomas (not shown).

Furthermore, collectively, the carcinomas harboring a mutation in any of the studied genes exhibited a higher Weiss score $(P=0.032)$ than wild-type carcinomas.

\section{Discussion}

The genetic background of adrenocortical carcinoma is not completely characterized. Several reports have identified activating $R A S$ mutations (Yashiro et al. 1994, Lin et al. 1998), which suggest activation of the Ras/Raf/MEK/ERK pathway. In the present study, in a panel of 35 archival formalin-fixed, paraffin-embedded adrenocortical tumor specimens, we detected two $B R A F$ mutations (5.7\%). Four tumor specimens $(11.4 \%)$ carried EGFR TK domain mutations. One specimen carried a KRAS mutation, and another carried two NRAS mutations. No mutations were identified in the two cell lines investigated.

Activating BRAF mutations have been detected in several types of malignancies. Malignant melanomas and papillary thyroid carcinomas have a particularly high frequency of activating V600E BRAF mutations, as well as a high frequency of $R A S$ mutations, although $B R A F$ and $R A S$ mutations appear to be mutually exclusive (Kimura et al. 2003, Garnett \& Marais 2004). The presence of those $B R A F$ and $R A S$ mutations confirms the importance of the Ras/Raf/MEK/ERK pathway for these malignancies and has been postulated to be linked to the ability of the tropic hormones MSH and TSH respectively, to activate an adenylate cyclase/cAMP/Ras/Raf-dependent signaling pathway that leads to cell proliferation (Garnett \& Marais 2004). Based on this hypothesis, BRAF and RAS mutations might also be anticipated in malignant adrenal cortex cells, because their normal counterparts also respond to angiotensin II by the activation of adenylate cyclase, elevation of intracellular cAMP levels, and activation of Ras, Raf-1, and MAPK (Smith et al. 1999). In our panel of adrenocortical carcinomas, we detected one V600E and one G464E BRAF mutation, both of which have previously reported in other malignancies and are known to have an activating effect. The presence of activating $B R A F$ mutations in malignant melanomas and thyroid carcinomas raises the possibility of future tailored targeted therapy with B-Raf inhibitors in patients selected based on this genotype, and such clinical trials are ongoing (www.clinicaltrials.gov). Such an inhibitor would also be a reasonable investigational option for the subset of adrenocortical carcinoma patients with $B R A F$ mutations that we detected.

EGFRIs such as gefitinib and erlotinib have clinical activity in the subset of NSCLC patients (around 10\% in US patients and $27.5 \%$ in Japanese patients; Fukuoka et al. 2003, Herbst et al. 2004) that harbor somatic mutations in the EGFR TK domain (Lynch et al. 2004, Paez et al. 2004, Pao et al. 2004). Such mutations promote EGF-induced receptor autophosphorylation, activate downstream signaling pathways and promote resistance to conventional chemotherapy (Sordella et al. 2004). We detected four tumor specimens harboring TK domain mutations in a panel of 35 adrenocortical carcinomas (11.4\%). This frequency is similar to that for EGFR TK domain mutations in NSCLCs in US patients, and higher than what we have previously found in another endocrine malignancy, namely thyroid carcinomas (3.2\% (Mitsiades et al. 2006)). One of these specimens harbored a G2193A transition resulting in a premature stop at codon 731, a mutation previously described in NSCLC (Huang et al. 2004). It is unclear how a truncated kinase domain could activate downstream signaling. However, it is possible that heterodimerization of the truncated EGFR with a full-length member of the HER family may result in a conformation that activates downstream signaling. In fact, another 

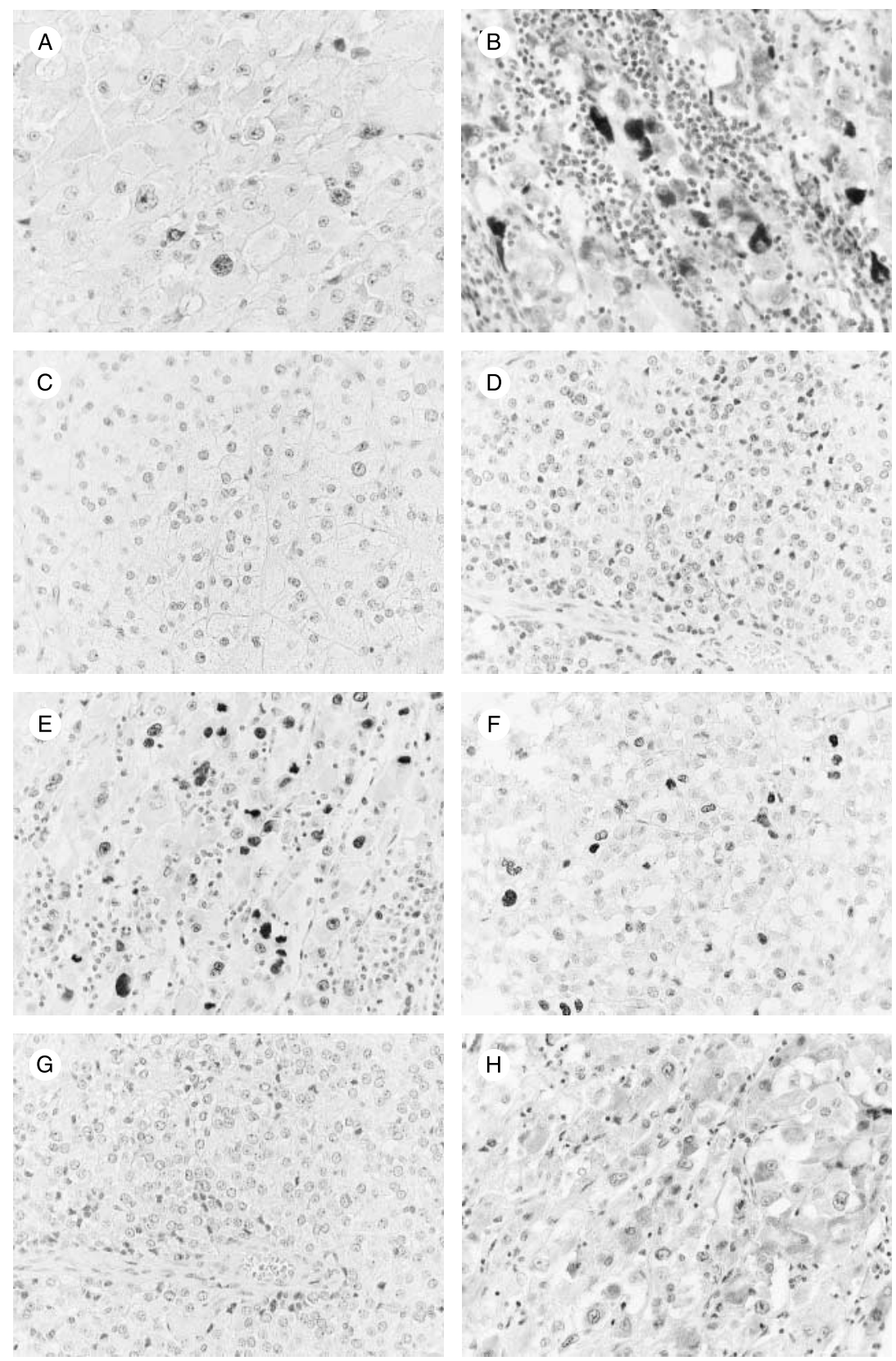

Figure 2 Representative examples of pEGFR (Tyr992), pMEK, and pMAPK immunostaining in adrenal carcinomas and adenomas. A-B. Immunostaining for pEGFR (Tyr992) was absent in an adrenal carcinoma that is wild-type for EGFR (A), and strong in a carcinoma carrying an EGFR mutation (B). C-F. Immunostaining for pMEK was totally absent in an adrenal adenoma (C), weak but detectable in an adrenal carcinoma that is wild-type for both $B R A F$ and $E G F R(D)$, and stronger in an adrenal carcinoma carrying a G464E BRAF mutation (E) and in an adrenal carcinoma carrying a F723S EGFR mutation (F). G-H. Immunostaining for phosphorylated MAPK (p44/42, ERK1/2) was very weak to totally absent in an adrenal carcinoma that is wild-type for both $B R A F$ and $\operatorname{EGFR}(\mathrm{G})$, and stronger in an adrenal carcinoma carrying a G464E BRAF mutation $(\mathrm{H})$. Magnifications: $\times 200$.

member of the HER family, HER3, that lacks entirely a functional kinase domain is known to promote growth and survival via heterodimerization with HER1 (EGFR) and HER2, leading to the activation of their (intact) kinase domains (Hsieh \& Moasser 2007).
Adrenocortical carcinomas harboring an EGFR mutation exhibited increased phosphorylation of EGFR (Tyr 992) compared with wild-type carcinomas. We also found that adrenocortical carcinomas with a BRAF or EGFR mutation exhibited stronger 
immunoreactivity for the phosphorylated forms of the kinases MEK and ERK, suggesting that these mutations activate this signaling pathway. It should be noted that, we documented immunohistochemically that all our adrenocortical carcinomas were strongly positive for Melan-A and negative for TTF-1, thus confirming their adrenal origin and ruling out the possibility of undetected metastasis from a lung carcinoma.

Our study involved a relatively large number of adrenocortical carcinomas, considering the rarity of this malignancy. A limitation of our study is that the tumor specimens were collected retrospectively from multiple institutions, from patients who had not been treated uniformly, and with limited clinical data available. As a result, we cannot assess the impact of these mutations on the clinical outcome. In any case, the small number of mutations found does not allow statistical analysis of the results, e.g., the immunohistochemical results. Furthermore, as both adrenocortical cell lines studied were wild-type for all genes examined, they cannot serve as a model for mechanistic experiments (e.g., to assess the impact of the mutation on enzymatic activity, cell growth or apoptosis or the effect of specific kinase inhibitors on cell biology). Moreover, prior experience in many tumor cell types suggests that these mutations are somatic, although we were unable to specifically demonstrate that in our panel, due to the lack of adequate normal tissue in most stored tumor specimens.

Effective systemic therapies for metastatic adrenocortical carcinoma are needed. As we have now entered the era of tailored targeted anticancer therapies, treatment decisions in several types of cancer are increasingly based on careful patient selection by tumor genotyping. For this approach to be applied in adrenocortical carcinomas, a detailed knowledge of their genetic background is necessary. Our study identifies mutations in the Ras/Raf/ MEK/ERK pathway and EGFR in a subset of adrenocortical carcinomas.

\section{Declaration of interest}

The authors have no conflict of interest to declare.

\section{Funding}

Work supported by PENED03/583 (research project by the Greek Secretariat for Research and Technology) awarded to V K.

\section{References}

Brose MS, Volpe P, Feldman M, Kumar M, Rishi I, Gerrero R, Einhorn E, Herlyn M, Minna J, Nicholson A et al. 2002 BRAF and RAS mutations in human lung cancer and melanoma. Cancer Research 62 6997-7000.

Davies H, Bignell GR, Cox C, Stephens P, Edkins S, Clegg S, Teague J, Woffendin H, Garnett MJ, Bottomley W et al. 2002 Mutations of the BRAF gene in human cancer. Nature 417 949-954.

Edgren M, Eriksson B, Wilander E, Westlin JE, Nilsson S \& Oberg K 1997 Biological characteristics of adrenocortical carcinoma: a study of p53, IGF, EGF-r, Ki-67 and PCNA in 17 adrenocortical carcinomas. Anticancer Research 17 1303-1309.

Fagin JA 2004 Challenging dogma in thyroid cancer molecular genetics - role of RET/PTC and BRAF in tumor initiation. Journal of Clinical Endocrinology and Metabolism 89 4264-4266.

Fukuoka M, Yano S, Giaccone G, Tamura T, Nakagawa K, Douillard JY, Nishiwaki Y, Vansteenkiste J, Kudoh S, Rischin D et al. 2003 Multi-institutional randomized phase II trial of gefitinib for previously treated patients with advanced non-small-cell lung cancer (The IDEAL 1 Trial) [corrected]. Journal of Clinical Oncology 21 2237-2246.

Garnett MJ \& Marais R 2004 Guilty as charged; B-RAF is a human oncogene. Cancer Cell 6 313-319.

Herbst RS, Fukuoka M \& Baselga J 2004 Gefitinib - a novel targeted approach to treating cancer. Nature Reviews. Cancer 4 956-965.

Houben R, Becker JC, Kappel A, Terheyden P, Brocker EB, Goetz R \& Rapp UR 2004 Constitutive activation of the Ras-Raf signaling pathway in metastatic melanoma is associated with poor prognosis. Journal of Carcinogenesis 36.

Hsieh AC \& Moasser MM 2007 Targeting HER proteins in cancer therapy and the role of the non-target HER3. British Journal of Cancer 97 453-457.

Huang SF, Liu HP, Li LH, Ku YC, Fu YN, Tsai HY, Chen YT, Lin YF, Chang WC, Kuo HP et al. 2004 High frequency of epidermal growth factor receptor mutations with complex patterns in non-small cell lung cancers related to gefitinib responsiveness in Taiwan. Clinical Cancer Research 10 8195-8203.

Ichihara S, Toyooka S, Fujiwara Y, Hotta K, Shigematsu H, Tokumo M, Soh J, Asano H, Ichimura K, Aoe K et al. 2007 The impact of epidermal growth factor receptor gene status on gefitinib-treated Japanese patients with non-small-cell lung cancer. International Journal of Cancer 120 1239-1247.

Kamio T, Shigematsu K, Sou H, Kawai K \& Tsuchiyama H 1990 Immunohistochemical expression of epidermal growth factor receptors in human adrenocortical carcinoma. Human Pathology 21 277-282.

Kimura ET, Nikiforova MN, Zhu Z, Knauf JA, Nikiforov YE \& Fagin JA 2003 High prevalence of BRAF mutations in 
thyroid cancer: genetic evidence for constitutive activation of the RET/PTC-RAS-BRAF signaling pathway in papillary thyroid carcinoma. Cancer Research 63 1454-1457.

Lin SR, Tsai JH, Yang YC \& Lee SC 1998 Mutations of $\mathrm{K}$-ras oncogene in human adrenal tumours in Taiwan. British Journal of Cancer 77 1060-1065.

Lynch TJ, Bell DW, Sordella R, Gurubhagavatula S, Okimoto RA, Brannigan BW, Harris PL, Haserlat SM, Supko JG, Haluska FG et al. 2004 Activating mutations in the epidermal growth factor receptor underlying responsiveness of non-small-cell lung cancer to gefitinib. New England Journal of Medicine 350 2129-2139.

Mitsiades CS, Kotoula V, Poulaki V, Sozopoulos E, Negri J, Charalambous E, Fanourakis G, Voutsinas G, TseleniBalafouta S \& Mitsiades N 2006 Epidermal growth factor receptor as a therapeutic target in human thyroid carcinoma: mutational and functional analysis. Journal of Clinical Endocrinology and Metabolism 91 3662-3666.

Mitsiades CS, Negri J, McMullan C, McMillin DW, Sozopoulos E, Fanourakis G, Voutsinas G, TseleniBalafouta S, Poulaki V, Batt D et al. 2007 Targeting BRAFV600E in thyroid carcinoma: therapeutic implications. Molecular Cancer Therapeutics 6 1070-1078.

Moroni M, Veronese S, Benvenuti S, Marrapese G, SartoreBianchi A, Di Nicolantonio F, Gambacorta M, Siena S \& Bardelli A 2005 Gene copy number for epidermal growth factor receptor (EGFR) and clinical response to antiEGFR treatment in colorectal cancer: a cohort study. Lancet Oncology 6 279-286.

Nikiforova MN, Kimura ET, Gandhi M, Biddinger PW, Knauf JA, Basolo F, Zhu Z, Giannini R, Salvatore G, Fusco A et al. 2003 BRAF mutations in thyroid tumors are restricted to papillary carcinomas and anaplastic or poorly differentiated carcinomas arising from papillary carcinomas. Journal of Clinical Endocrinology and Metabolism 88 5399-5404.

Paez JG, Janne PA, Lee JC, Tracy S, Greulich H, Gabriel S, Herman P, Kaye FJ, Lindeman N, Boggon TJ et al. 2004 EGFR mutations in lung cancer: correlation with clinical response to gefitinib therapy. Science 304 1497-1500.
Pao W, Miller V, Zakowski M, Doherty J, Politi K, Sarkaria I, Singh B, Heelan R, Rusch V, Fulton L et al. 2004 EGF receptor gene mutations are common in lung cancers from 'never smokers' and are associated with sensitivity of tumors to gefitinib and erlotinib. PNAS 101 13306-13311.

Pollock PM \& Meltzer PS 2002 A genome-based strategy uncovers frequent BRAF mutations in melanoma. Cancer Cell 2 5-7.

Rajagopalan H, Bardelli A, Lengauer C, Kinzler KW, Vogelstein B \& Velculescu VE 2002 Tumorigenesis: RAF/RAS oncogenes and mismatch-repair status. Nature 418934.

Smith RD, Baukal AJ, Dent P \& Catt KJ 1999 Raf-1 kinase activation by angiotensin II in adrenal glomerulosa cells: roles of $\mathrm{Gi}$, phosphatidylinositol 3-kinase, and $\mathrm{Ca}^{2+}$ influx. Endocrinology 140 1385-1391.

Sordella R, Bell DW, Haber DA \& Settleman J 2004 Gefitinib-sensitizing EGFR mutations in lung cancer activate anti-apoptotic pathways. Science 305 1163-1167.

Suzuki Y, Orita M, Shiraishi M, Hayashi K \& Sekiya T 1990 Detection of ras gene mutations in human lung cancers by single-strand conformation polymorphism analysis of polymerase chain reaction products. Oncogene $\mathbf{5}$ 1037-1043.

Tam IY, Chung LP, Suen WS, Wang E, Wong MC, Ho KK, Lam WK, Chiu SW, Girard L, Minna JD et al. 2006 Distinct epidermal growth factor receptor and KRAS mutation patterns in non-small cell lung cancer patients with different tobacco exposure and clinicopathologic features. Clinical Cancer Research 12 1647-1653.

Volante M, Buttigliero C, Greco E, Berruti A \& Papotti M 2008 Pathological and molecular features of adrenocortical carcinoma: an update. Journal of Clinical Pathology 61 787-793.

Yashiro T, Hara H, Fulton NC, Obara T \& Kaplan EL 1994 Point mutations of ras genes in human adrenal cortical tumors: absence in adrenocortical hyperplasia. World Journal of Surgery 18 455-460. 